\title{
DESENVOLVIMENTO E CARACTERIZAÇÃO DE BOLDO SOLUÇÃO ORAL EM INDÚSTRIA FARMACÊUTICA PERNAMBUCANA
}

\author{
I. C. M. MAIA ${ }^{1}$, G. S. A. LIMA ${ }^{1}$, T. P. SOUZA $^{2,4}$, A. V. GUEDES ${ }^{3,4}$, J. L.S. SOBRINHO ${ }^{1}$, \\ M. F. R. SOARES ${ }^{1}$, J. L. SILVA ${ }^{4}$
}

${ }^{1}$ Universidade Federal de Pernambuco, Centro de Ciências da Saúde, Departamento de Ciências Farmacêuticas, Núcleo de Controle de Qualidade de Medicamentos e Correlatos (NCQMC)

${ }^{2}$ Faculdades Integradas de Vitória de Santo Antão, Departamento de Farmácia

${ }^{3}$ Universidade Federal de Pernambuco, Centro Acadêmico de Vitória

${ }^{4}$ Lapon Industria Farmacêutica LTDA

E-mail para contato: iaramilanez14@gmail.com

RESUMO - Fitoterápicos, são produtos industrializados oriundos de material vegetal, e estão ganhando cada vez mais visibilidade e força no Brasil, alguns autores demonstram que o boldo é uma das plantas medicinais mais utilizadas popularmente em diversas regiões do Brasil. O presente artigo pretende elucidar o desenvolvimento de uma solução oral a partir de extrato padronizado de Boldo (Peumus boldus) 0,1\%. A solução foi preparada com a diluição do extrato de boldo 0,1\% em glicerina; metilparabeno e propilparabeno, álcool etílico e corante caramelo. A caracterização do produto foi realizada pelos testes de identificação e doseamento; ambos se deram conforme monografia da Farmacopeia Brasileira $5^{a}$ edição. A identificação foi por cromatografia em camada delgada (CCD), utilizando $2 \mathrm{mg}$ de Boldina SQR como referência. A visualização foi na luz UV a $365 \mathrm{~nm}$ e em luz visivel. O doseamento de alcaloides totais da solução foi expresso em boldina. Nos resultados da identificação, foi possível visualizar as bandas correspondentes à Boldina na Solução Oral de Boldo, tanto em UV quanto na luz visivel. O teor de alcaloides totais expressos em boldina encontrados estavam dentro do intervalo preconizado pela monografia farmacopêica. Nessas condições, o produto Boldo solução oral, cumpre os requisitos de segurança e eficácia necessários para prosseguir com o seu desenvolvimento e posterior entrada no mercado como medicamento tradicional fitoterápico de Registro Simplificado.

Palavras-Chave: boldo. boldina. fitoterápicos. indústria farmacêutica.

ABSTRACT - Phytoterapics, are industrialized products derived from plant and are gaining more visibility and strength in Brazil, some authors demonstrate that the boldo is one of the medicinal plants most popularly used in several regions of Brazil. The present article intends to elucidate the development of an oral solution from standard extract of Boldo (Peumus boldus) $0,1 \%$. The solution was prepared with the dilution of the $0.1 \%$ boldo extract in glycerin; methylparaben and 
propylparaben and caramel coloring. The characterization of the product were performed by identification and dosing methods, both

following the Brazilian Pharmacopoeia fifth edition monograph, and were performed in triplicate. The identification were performed by thin-layer chromatography (TLC) method, using $2 \mathrm{mg}$ of Boldine CRS as reference. The visualization were in both $U V$ and visible light. The dosing test used total alkaloids expressed as boldine. In the identification results, it was possible to visualize the bands corresponding to Boldine in the Boldo Oral Solution, in both UV and visible light. The content of total alkaloids expressed as boldine was within the range recommended by ANVISA. By all means, the Boldo oral solution meets the safety and efficacy requirements necessary to proceed with its development and subsequent entry into the consumer market.

Key words: boldo. boldine. phytotherapic drugs. pharmaceltical industry.

\section{INTRODUÇÃO}

Desde que se reconhece a existência da medicina, as plantas medicinais desempenham seu papel na busca da cura para os males que acometem o ser humano (BRASIL, 2006). Fitoterápicos, são produtos industrializados oriundos de material vegetal, e estão ganhando cada vez mais visibilidade e força no Brasil (SANTOS et al., 2017). Com o intuito de disseminar e ao mesmo tempo regulamentar terapias alternativas, o Ministério da Saúde criou a Política Nacional de Práticas Integrativas e Complementares (PNPIC), vinculada ao Sistema Único de Saúde (SUS), que busca incluir a utilização de plantas medicinais e fitoterápicos no sistema. O PNPIC garante aos usuários do SUS acesso gratuito à fitoterápicos, enquanto usados de forma racional e incentiva a pesquisa e desenvolvimento de novos fitoterápicos a partir da biodiversidade brasileira.

A Peumus boldus Molina, com nome popular Boldo ou Boldo-do-Chile, foi identificada como planta de uso medicinal devido a achados em sítios arqueológicos chilenos de cerca de 12 mil anos atrás. Estudos recentes (MACEDO et al., 2007; GRIZ et al., 2017) demonstram que o boldo é uma das plantas medicinais mais utilizadas popularmente em diversas regiões do Brasil. De acordo com um estudo realizado por Caetano et al. (2015), suas indicações terapêuticas estão relacionadas principalmente a problemas no trato gastrointestinal e hepático, como dor no estômago, gastrite, prisão de ventre e azia. Peumus boldus Molina consta hoje na Lista de Medicamentos Fitoterápicos de Registro Simplificado, da ANVISA (BRASIL, 2008). A forma de apresentação da droga vegetal pode ser em extrato ou tintura das folhas da planta. Uma das substâncias responsável pelos efeitos terapêuticos do extrato de folhas de Peumus boldus Molina é a boldina, um alcaloide específico dessa espécie de planta. Diversos estudos atribuem à boldina os efeitos farmacológicos obtidos com a administração oral do extrato padronizado do Boldo. Estudos in vivo mostram que a boldina é rapidamente absorvida e fica preferencialmente acumulada no fígado (ESCOP, 2003).

Mantendo em mente a capacidade do Brasil no desenvolvimento de novos fitoterápicos e na descoberta de novas plantas medicinais devido à sua grande biodiversidade, a indústria farmacêutica surge nesse cenário desejando atender a demanda do público por esses produtos de origem natural. Segundo HASENCLEVER et al. (2017), uma das maiores dificuldades para a indústria se inserir nesse meio é a caracterização da 
matéria prima e dos produtos publicados na Lista de Medicamentos Fitoterápicos de Registro Simplificado (BRASIL, 2008). Sendo assim, o objetivo do presente trabalho é o desenvolvimento de uma solução oral a partir de extrato padronizado de boldo em uma indústria farmacêutica pernambucana.

\section{MATERIAIS E MÉTODOS}

\subsection{Equipamentos utilizados}

Banho- Maria Marconi MA-127, Papel de filtro Unifil faixa preta C41, Balança analítica HR 200, Placa de sílica gel, Merck (GF254) com fluorescência, Lâmpada Ultravioleta CAM-001, Borrifador plástico, Cuba cromatográfica, Capela de exaustão, Evaporador rotatório QUIMIS, pHmetro HANNA HI 221, Picnômetro Vidrolabor 25 mL, Funil de separação Vidrolabor 250 mL. Substâncias utilizadas: Boldina SQR - Código: Y0000434; lote:3 - Farmacopeia Europeia, Extrato fluído padronizado Peumus boldus 0,1\% Laboratório Catedral, Boldo solução oral, Glicerina, Metilparabeno, Propilparabeno, Corante Caramelo, Sulfato de Sódio Anidro. Solventes utilizados: Tolueno Synth, Metanol Vertec Química Fina, Dietilamina Química moderna, Éter etílico Química moderna, Álcool Etílico, Diclorometano Vertec Química Fina. Soluções utilizadas: Ácido clorídrico $2 \mathrm{M}$, Hidróxido de amônio 6M, Iodobismutato de potássio aquo-acético, Nitrito de sódio, Iodeto de potássio mercúrio SR, Ácido Sulfúrico 0,005M, Hidróxido de Sódio 0,01M, Vermelho de Metila SI.

\subsection{Procedimento formulação do produto}

O extrato fluido padronizado de Peumus boldus $0,1 \%$ e a glicerina foram misturados por 10 minutos em temperatura ambiente, em seguida dissolveu-se metilparabeno $\mathrm{e}$ propilparabeno em álcool etílico. Posteriormente, corante caramelo foi dissolvido em água purificada e misturado por 2 minutos, posteriormente adicionou-se água purificada e agitou-se a solução por mais 15 minutos. Por fim, a solução final permaneceu em repouso por 30 minutos. O pHmetro definiu o $\mathrm{pH}$ da solução, e a densidade foi estabelecida utilizando-se picnômetro de $25 \mathrm{~mL}$ previamente calibrado.

\subsection{Caracterização do produto}

Realizou-se a identificação do produto Boldo solução oral conforme Farmacopeia Brasileira $5^{\mathrm{a}}$ edição (BRASIL, 2010). As análises foram realizadas em triplicata para maior confiabilidade de resultados. Preparou-se a fase móvel utilizando tolueno, metanol e dietilamina na proporção (8:1:1), em seguida secou-se $25 \mathrm{ml}$ do produto em banho-maria a $60^{\circ} \mathrm{C}$ até completa evaporação dos solventes, adicionou-se $10 \mathrm{~mL}$ de ácido clorídrico $2 \mathrm{M}$ e em seguida, o resíduo foi filtrado com papel de filtro e alcalinizado com hidróxido de amônio $6 \mathrm{M}$ até atingir $\mathrm{pH}$ 9. A solução foi transferida para funil de separação e realizouse a extração com $20 \mathrm{~mL}$ de éter etílico em duplicata. Em seguida, as fazes orgânicas foram reunidas e filtradas em papel de filtro, A fase orgânica foi secada até resíduo, em banhomaria a $60^{\circ} \mathrm{C}$. Suspendeu-se o resíduo em $1 \mathrm{~mL}$ de metanol. O mesmo procedimento descrito acima foi utilizado para identificação do extrato fluido. A solução referência foi preparada pesando-se $2 \mathrm{mg}$ de bodina SQR em seguida diluiu-se a mesma em $5 \mathrm{~mL}$ de metanol. Após o preparo das soluções desenvolveu-se o cromatograma: Aplicou-se na cromatoplaca, separadamente, em forma de banda, $20 \mu \mathrm{L}$ da Solução amostra do produto, $20 \mu \mathrm{L}$ da solução amostra de extrato fluido e $20 \mu \mathrm{L}$ da solução referência. Desenvolveuse o cromatograma. Posteriormente a placa foi removida da fase móvel e seca ao ar, após a secagem foi examinada sob luz ultravioleta em $365 \mathrm{~nm}$. Logo em seguida a placa foi 
nebulizada com iodobismutato de potássio aquo-acético e secou por 5 minutos, posteriormente foi novamente nebulizada com nitrito de sódio solução reagente. Após 30 minutos foi a placa foi observada sob luz visível. E por fim calculou-se os valores de Rf do SQR, extrato padronizado e boldo solução oral.

O doseamento de alcaloides totais do produto Boldo Solução oral foi realizado de acordo com a Farmacopeia Brasileira $5^{\text {a }}$ Edição (BRASIL, 2010). As análises foram realizadas em triplicata para maior confiabilidade de resultados. $100 \mathrm{~g}$ de Boldo Solução oral foram pesados e em seguida, evaporados em evaporador rotatório até consistência de extrato mole. Transferiu-se quantitativamente a amostra para um funil de separação com $5 \mathrm{~mL}$ de água. Adicionou-se $6 \mathrm{~mL}$ de hidróxido de amônio $6 \mathrm{M}$, seguido de agitação com sucessivas frações de diclorometano. Verificou-se a completa extração dos alcaloides adicionando uma gota de iodeto de potássio mercúrio SR a algumas gotas da fase aquosa. As fases orgânicas foram reunidas em funil de separação e lavadas com água até neutralização. Foram adicionados $2 \mathrm{~g}$ de sulfato de sódio anidro, deixados em contato com a fase orgânica por alguns minutos com agitações casuais. O sulfato de sódio foi decantado e filtrado com $10 \mathrm{~mL}$ de diclorometano três vezes. As fases orgânicas foram reunidas e evaporadas em evaporador rotatório. Ao resíduo final, adicionou-se $20 \mathrm{~mL}$ de ácido sulfúrico $0,005 \mathrm{M} \mathrm{SV}$. A solução final foi titulada com hidróxido de sódio $0,01 \mathrm{M} \mathrm{SV}$ em presença de vermelho de metila SI. O mesmo procedimento descrito acima foi utilizado para doseamento de alcaloides do extrato fluido.

\section{RESULTADOS E DISCUSSÃO}

\subsection{Formulação}

Obteve-se uma solução límpida de coloração castanho claro isenta de partículas estranhas com sabor levemente adocicado e característico de boldo. $\mathrm{O} \mathrm{pH}$ obtido foi de 5,4 , sendo que os valores de padrão interno da indústria são entre 4 e 6 , a densidade obtida foi de $0,98 \mathrm{~g} / \mathrm{ml}$, este valor também se encontra de acordo com as determinações internas da indústria que são de 0,9 a $1,1 \mathrm{~g} / \mathrm{ml}$.

\subsection{Caracterização do Produto}

Foram visualizados, sob a luz UV no comprimento de onda $365 \mathrm{~nm}$, bandas dos padrões de referência de boldina, todas as placas apresentando uma mancha azul violácea iguais em posição, tanto no extrato de boldo como no produto acabado Boldo Solução Oral conforme mostra na Figura 01 e 02. Após nebulização com reagentes reveladores, foi possível observar sob luz visível, bandas dos padrões de referência de boldina, todas as placas apresentando uma mancha castanha, tanto no extrato de boldo quanto no produto acabado, Boldo Solução oral conforme mostra na Figura 03 e 04 . O valor de Rf encontrado foi de 0,53 tanto para o SQR quanto para o extrato padronizado de boldina e para o boldo solução oral, apesar de não descrito na farmacopeia o valor de Rf da boldina encontrado é equivalente ao apresentado por Simões (2016). Os resultados obtidos na cromatografia de camada delgada (CCD) corroboram com o resultado apresentado por Santana (2007) e estão de acordo com o que preconiza a Farmacopeia Brasileira $5^{\text {a }}$ Edição (BRASIL,2010). Com o resultado da identificação, é possível confirmar a presença de boldina no produto final Boldo Solução oral, garantindo assim sua eficácia farmacológica já que a boldina é o alcaloide principal responsável pelo efeito colerético, que é o aumento da secreção de bile, descongestionando o fígado e favorecendo a digestão, de acordo com SAAD (2009). 
Figura 1: Placas CCD, revelação em comprimento de onda UV 365 nm: 1- boldina; 2extrato fluido de boldo $0,1 \%$.
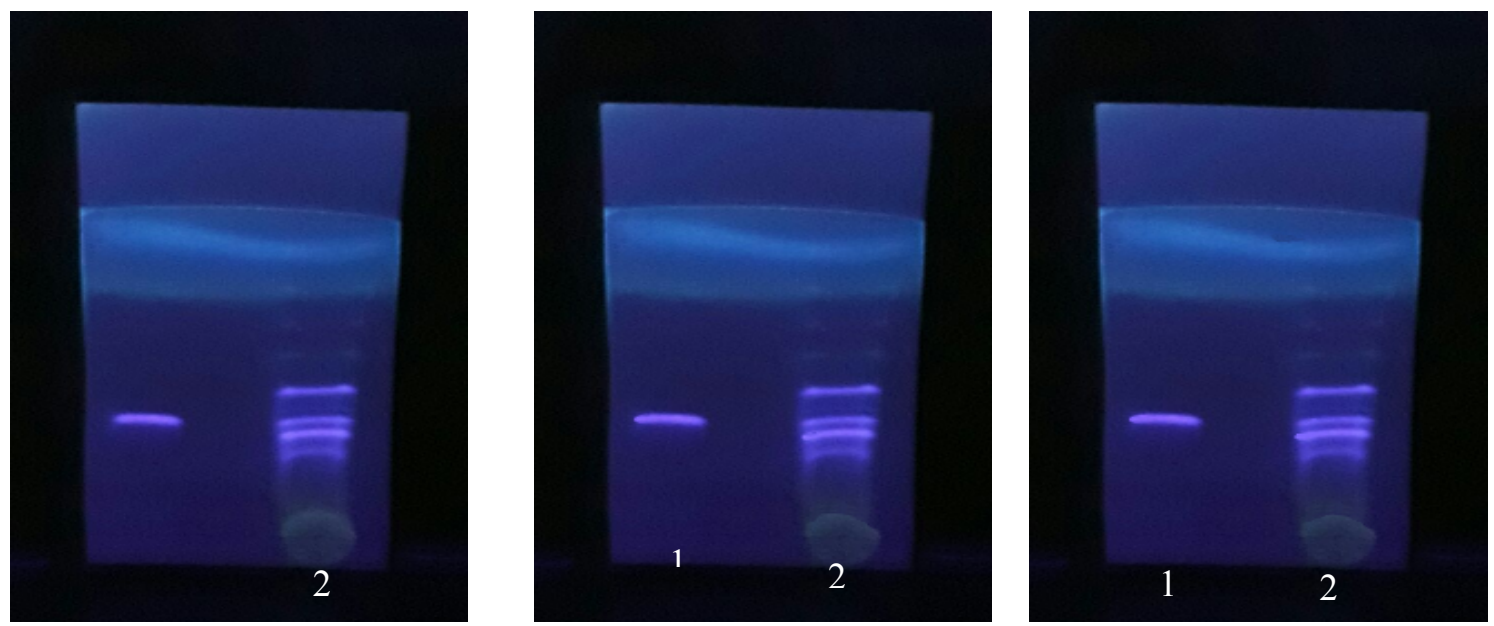

Figura 2: Placas CCD, revelação em comprimento de onda UV 365 nm: 1- boldina; 2Boldo solução oral.
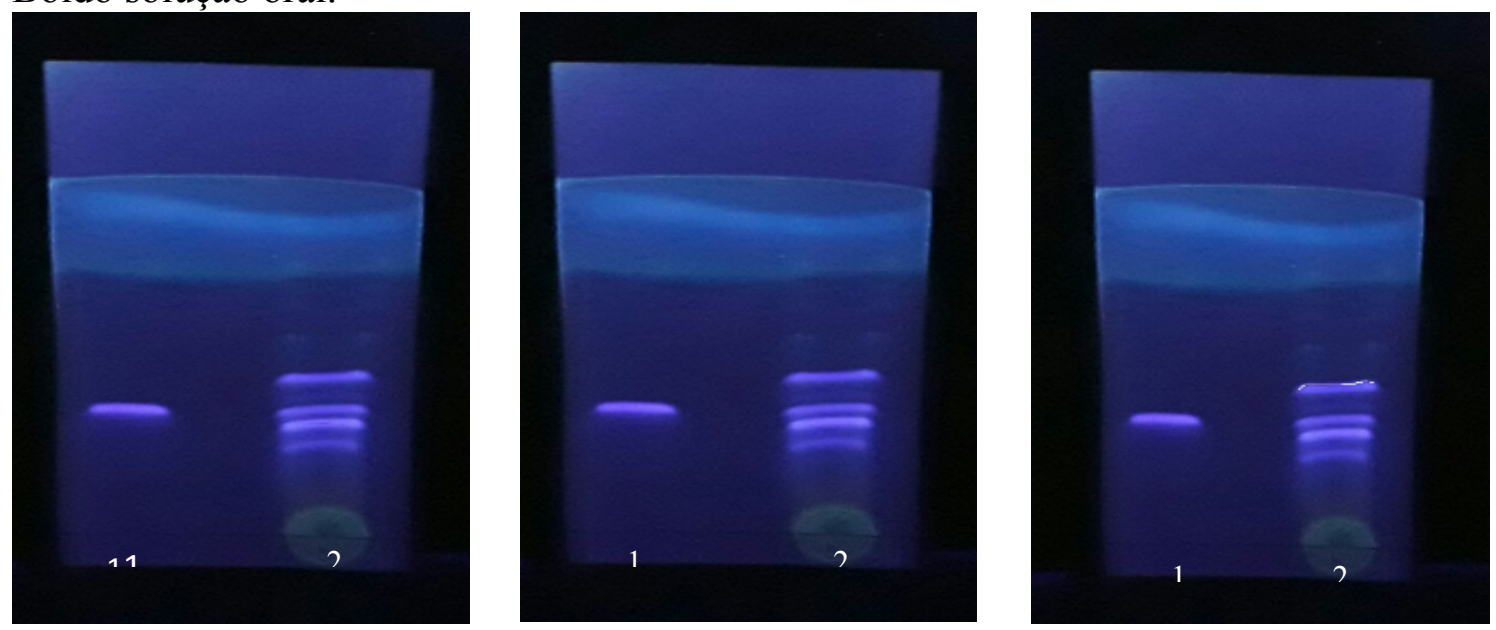
Figura 3: Placas CCD, revelação sob luz visível: 1- boldina; 2- extrato fluido de boldo $0,1 \%$.
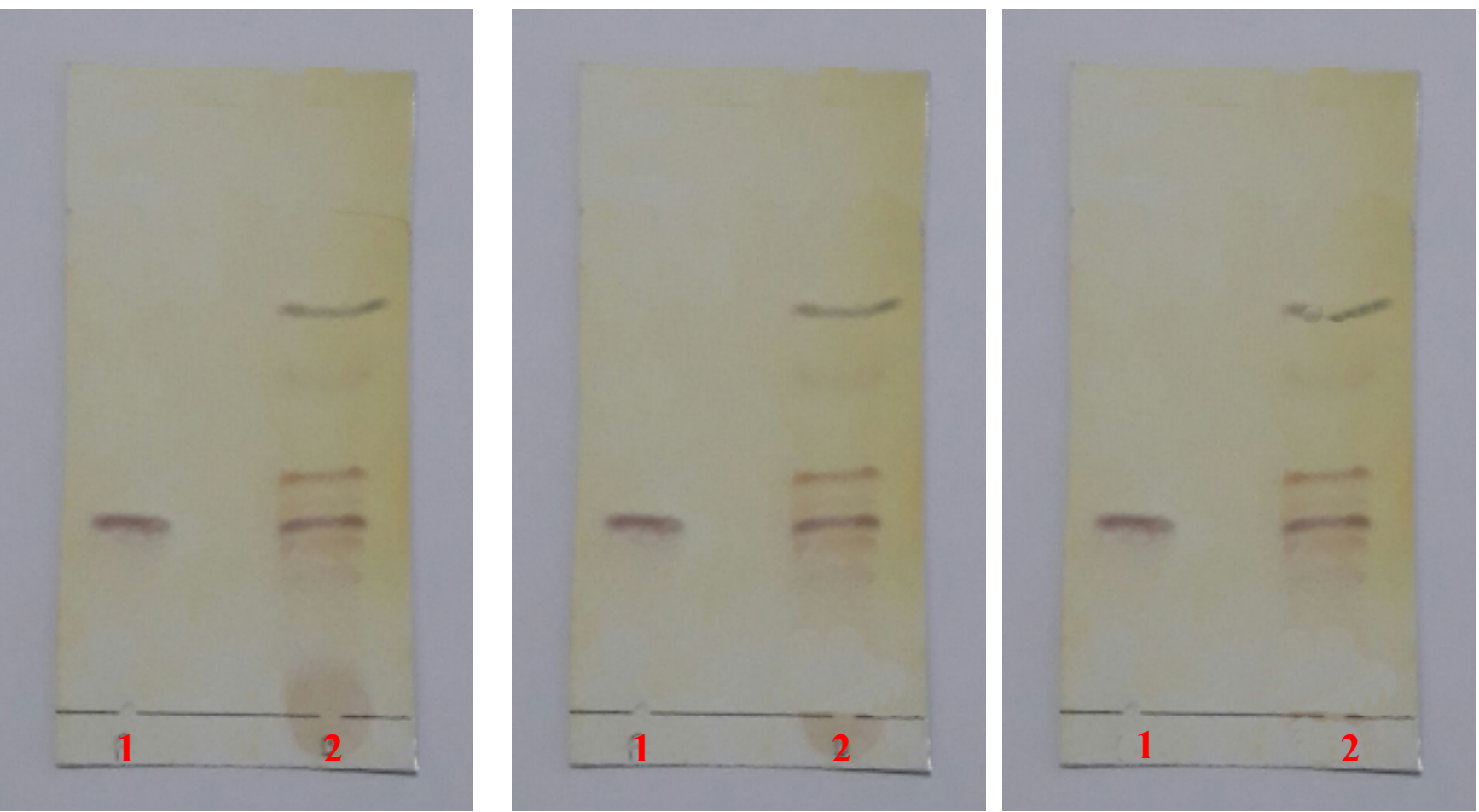

Figura 4: Placas CCD, revelação sob luz visível: 1- boldina; 2- Boldo solução oral.
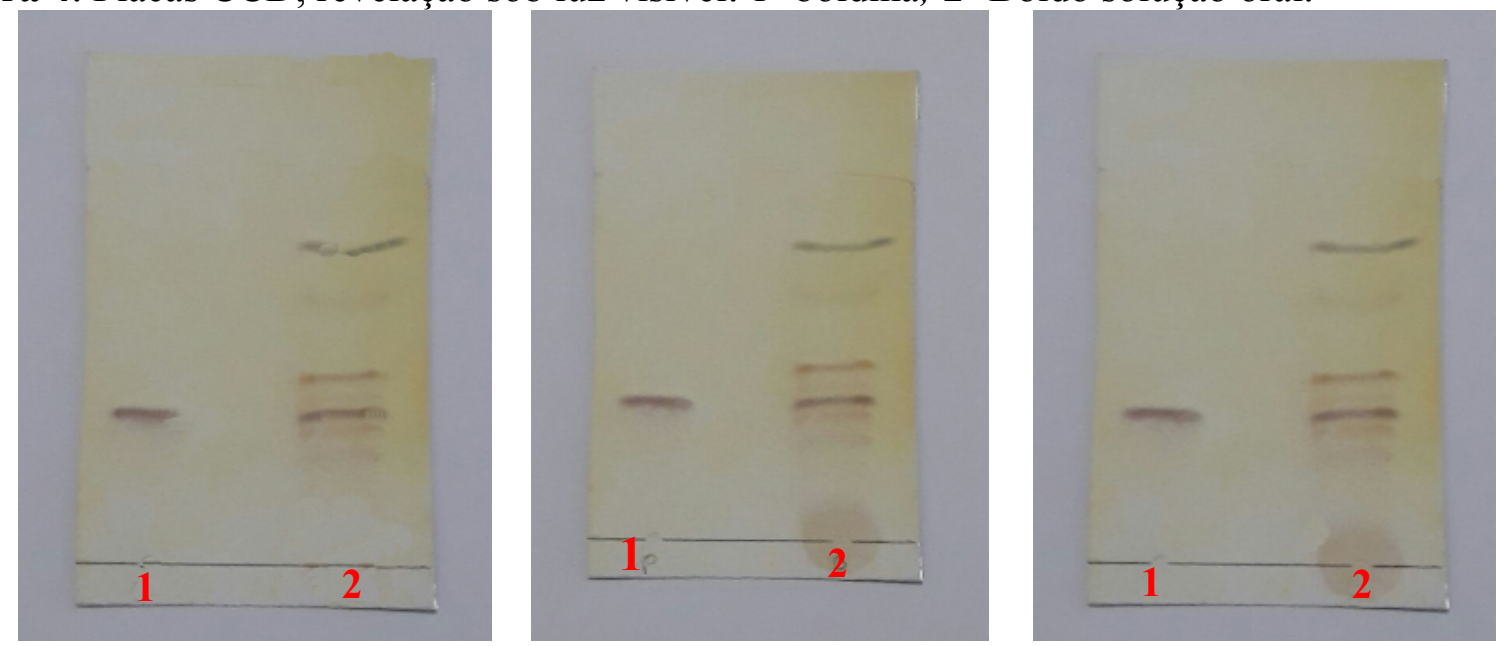

O doseamento foi realizado segundo a Farmacopeia Brasileira $5^{\text {a }}$ Edição (BRASIL, 2010), o teor de boldina foi definido pelas especificações internas da indústria de acordo com pesquisa de mercado, onde foi definido um teor de alcaloides totais, expressos em boldina de $0,0067 \%$ e o extrato fluido possui uma concentração esperada de $0,1 \%$. De acordo com o preconizado pela RDC 166/17 (BRASIL,2017) aceita-se uma variação de \pm $10 \%$ em ambos os casos. Após o procedimento realizado, obteve-se o teor de boldina total do produto Boldo Solução oral e do Extrato Fluido de acordo com a seguinte fórmula:

$$
T(\%)=\underline{32.74 \times(20-n)}
$$

$10 n-\frac{10}{20}$

Onde: $n=$ número de mililitros de hidróxido de sódio 0,01 M SV gastos; 
$m=$ massa do extrato, em $\mathrm{g}$;

$\mathrm{T}(\%)=$ Teor de alcaloides totais, expressos em boldina.

Os resultados apresentados na Tabela 1 mostram que todas as análises realizadas apresentam média dentro da faixa de aceitação tanto para o extrato quanto para o produto em teste, o desvio padrão apresenta valores baixos e o coeficiente de variação apresenta resultados abaixo de 5\% assim como preconizado pela RDC 166/17 (BRASIL,2017).

Tabela 1 - Resultado de doseamento de alcaloides totais expressos em boldina.

\begin{tabular}{|c|c|c|c|c|c|}
\hline \multicolumn{2}{|c|}{ Análise } & Teor $(\%)$ & Média & Desvio padrão & Coeficiente de variação \\
\hline \multirow{3}{*}{ Extrato } & $1^{\mathrm{a}}$ & 0,101 & \multirow{3}{*}{0,1007} & \multirow{3}{*}{$\mathbf{0 , 0 0 2 5 1 7}$} & \multirow{3}{*}{2,50} \\
\hline & $2^{\mathrm{a}}$ & 0,103 & & & \\
\hline & $3^{\mathrm{a}}$ & 0,098 & & & \\
\hline \multirow{3}{*}{ Produto } & $1^{\mathrm{a}}$ & 0,0065 & \multirow{3}{*}{0,0065} & \multirow{3}{*}{0,000252} & \multirow{3}{*}{3,85} \\
\hline & $2^{\mathrm{a}}$ & 0,0068 & & & \\
\hline & $3^{\mathrm{a}}$ & 0,0063 & & & \\
\hline
\end{tabular}

De acordo com o resultado obtido, o teor de alcaloides totais expressos em boldina, está dentro dos valores estabelecidos pela indústria, segundo a pesquisa de produtos desenvolvida pelo $\mathrm{P} \& \mathrm{D}$, nessas concentrações o produto Boldo solução oral, cumpre os requisitos de segurança e eficácia necessários para prosseguir com o seu desenvolvimento e posterior entrada no mercado consumidor.

\section{CONCLUSÃO}

Visto que o Peumus boldus atualmente elenca a Lista de Medicamentos Fitoterápicos de Registro Simplificado, da ANVISA (BRASIL, 2008) e que suas propriedades terapêuticas já estão bastante elucidadas e de uso disseminado pela população em geral, é de grande interesse da indústria farmacêutica atender a demanda do público por produtos fitoterápicos. A formulação e as análises elucidadas foram realizadas em escala de bancada, visto que o produto em questão cumpriu todos as exigências estipuladas pelo $\mathrm{P} \& \mathrm{D}$ da indústria e em conformidade com a farmacopeia em vigência, a próxima etapa é transpor a escala para piloto e posteriormente para escala industrial. Vale lembrar que paralelo a este estudo vem sendo realizado um estudo de estabilidade do produto. De acordo com os resultados já obtidos, essa formulação se mostra muito promissora para elencar o quadro de produtos produzidos pelo Lapon Indústria Farmacêutica LTDA.

\section{REFERÊNCIAS}

BRASIL. ANVISA. Agência Nacional de Vigilância Sanitária. Farmacopeia Brasileira, 5 ed. Brasília, DF, v.2, p. 698, 2010.

BRASIL. ANVISA. Agência Nacional de Vigilância Sanitária. Instrução Normativa $n^{\circ} 5$ de 11 de Dezembro de 2008. Lista de medicamentos fitoterápicos de registro simplificado.

BRASIL. ANVISA. Agência Nacional de Vigilância Sanitária Resolução. RDC no 166, de 24 de Julho de 2017. "Dispõe sobre a validação de métodos analíticos e dá outras providências". Disponível em: <https://www20.anvisa.gov.br/coifa/pdf/rdc166.pdf $>$. Acessado em: 05/11/17. 
CAETANO, N.L.B.; FERREIRA, et al.; Medicinal Plants Used by the Population of Lagarto, SE, Brazil - Emphasis in Cancer Patients. Rev. bras. plantas med., v. 17, no. 4, p. 748-756, 2015.

EUROPEAN SCIENTIFIC COOPERATIVE ON PHYTOTHERAPY. The Scientific Foundation for Herbal Medicinal Products, New York: Thieme Editor, 2003.

GRIZ, S. A. S.; MATOS-ROCHA, T. J.; et al.; Medicinal plants profile used by the 3rd District population of Maceió-AL. Braz. J., v. 77, no. 4, p. 794-802, 2017.

HASENCLEVER, L.; PARANHOS, J.; REIS COSTA.; et al.; A indústria de fitoterápicos brasileira: desafios e oportunidades. Ciênc. saúde coletiva, v. 22, p. 2559-2569, 2017.

MACEDO, A.F.; OSHIIWA, M.; GUARIDO, C.F. Ocorrência do uso de plantas medicinais por moradores de um bairro do município de Marília - SP. Rev. ciênc. farm. básica apl., v. 28, no. 1, p. 123-128, 2007.

SAAD, G. A.; LÉDA, P. H. O.; SÁ, I. M.; SEIXLACK, A. C. C. Fitoterapia Contemporânea -Tradição e Ciência na Prática Clínica, Rio de Janeiro: Editora Elsevier, 2009.

SANTANA, I.G.; SEVERO, I. L.; et al.; Determinação do perfil cromatográfico de extratos secos vegetais. An. eletr. XV sem. científica farmacêutica., Goiânia: UFG, 2007. n.p.

SANTOS, R. S.; MOTA, L. H. S.; MARQUES, B. C.; et al.; Uso regular de plantas medicinais para fins terapêuticos em famílias residentes na zona rural de Santo Antônio de Jesus - Bahia - Brasil. J. Health Biol. Sci., v. 5, p. 354-370, 2017

SIMÕES C. M. O., SCHENKEL E. P., MELLO J. C. P., Farmacognosia: Do Produto Natural ao Medicamento, São Paulo: Atmed Editora, 2016.

WHO. World Health Organization. The World Traditional Medicines Situation, in Traditional medicines: Global Situation, Issues and Challenges. Geneva, p. 1-14, 2011. 